\title{
ON A COMPARISON RESULT FOR MARKOV PROCESSES
}

\author{
LUDGER RÜSCHENDORF, ${ }^{*}$ University of Freiburg
}

\begin{abstract}
A comparison theorem is stated for Markov processes in Polish state spaces. We consider a general class of stochastic orderings induced by a cone of real functions. The main result states that stochastic monotonicity of one process and comparability of the infinitesimal generators imply ordering of the processes. Several applications to convex type and to dependence orderings are given. In particular, Liggett's theorem on the association of Markov processes is a consequence of this comparison result.
\end{abstract}

Keywords: Association of Markov processes; stochastic monotonicity; infinitesimal generator; stochastic ordering

2000 Mathematics Subject Classification: Primary 60E15; 60J25

\section{Introduction}

This paper is motivated by Liggett's (1985) characterization of (positive) association in Markov processes, which is the main tool used to establish this strong dependence notion. This result is the basis of many important applications and it has been modified and extended in various ways. For its role in connection with several interesting models in applied probability, see, in particular, Szekli (1995, Chapter 3).

Liggett's theorem is based on the notion of stochastic monotonicity and on the infinitesimal generator $A$ of the Markov process $X$. The main result of this paper is the comparison of two Markov processes $X$ and $Y$ with respect to a general class of stochastic orderings ' $\prec \mathscr{F}$ ', induced by some cone $\mathcal{F}$ of real functions on the state space $E$. Stochastic monotonicity and ordering of the infinitesimal generators $A$ and $B$ are the basic ingredients of the comparison result.

Positive dependence of a random vector $Z=\left(Z_{1}, \ldots, Z_{d}\right)$ is typically defined by a comparison with its copy $Z^{\perp}$ with independent components with respect to some class of (positive) dependence functions. Therefore, as a consequence of the comparison result, we also obtain several results on positive dependence orderings. In particular, Liggett's association theorem is a consequence of this comparison result.

Ordering conditions for Markov processes in terms of infinitesimal generators have been given in several papers. Massey (1987), Herbst and Pitt (1991), Chen and Wang (1993), Chen (2004), and Daduna and Szekli (2006) described stochastic ordering for discrete-state spaces, for diffusions, and for diffusions with jumps in terms of generators. For bounded generators and in the case of discrete-state spaces, Daduna and Szekli (2006) gave a comparison result for stochastic ordering in terms of a comparison of the generators. For an infinite-dimensional system of interacting diffusions, a comparison result for directionally convex ordering has been established in Cox et al. (1996) and Greven et al. (2002) under the condition that the diffusion

Received 26 June 2007; revision received 21 November 2007.

* Postal address: Department of Mathematical Stochastics, University of Freiburg, Eckerstrasse 1, D-79104 Freiburg, Germany. Email address: ruschen@stochastik.uni-freiburg.de 
coefficients are comparable. For Lévy processes, Bäuerle et al. (2006) and Bergenthum and Rüschendorf (2007) derived a comparison of the supermodular as well as of further orderings in terms of the corresponding ordering of the infinitesimal generator.

The proof of the main comparison result in the present paper is given in the same framework as Liggett's result and uses a similar idea as in Liggett's characterization of association (see Szekli (1995, Chapter 3.7)). The same idea of proof has also been used before in the papers of Cox et al. (1996) and Greven et al. (2002) mentioned above for directionally convex ordering of interacting diffusions. The author of this paper is grateful to a reviewer for a hint to these papers.

Motivated by the comparison results for option prices, an alternative approach to comparison theorems has been developed based on stochastic analysis (Itô's formula and Kolmogorov's backward equation) which allows us to go beyond the frame of Markov processes to semimartingales (see Bergenthum and Rüschendorf (2007) for recent developments on this approach). For the case of Markov processes, the results of Bergenthum and Rüschendorf (2007) are comparable to the results of this paper. However, in comparison, the approach via generators in this paper is more direct and simple.

\section{The comparison result}

For a homogeneous Markov process $X=\left(X_{t}\right)_{t \geq 0}$ with values in a compact partially ordered set $E$, Liggett (1985) established an important criterion for the positive dependence notion of the association of $X_{t}, t \geq 0$. Let $X$ be a strongly continuous Feller process with corresponding semigroup $S=\left(S_{t}\right)_{t \geq 0}$ of transition operators on $C_{b}(E)$. Let $A$ denote the infinitesimal generator of $S$ with domain $D_{A}$. Then $D_{A} \cap \mathcal{F}_{i}^{+}$is dense in $\mathcal{F}_{i}^{+}$, the class of bounded, nondecreasing, nonnegative functions on $E$ (see Szekli (1995)).

We call $X$ associated in time if, for all $0 \leq t_{1}<\cdots<t_{k}$, the vector $\left(X_{t_{1}}, \ldots, X_{t_{k}}\right)$ is associated, i.e.

$$
\mathrm{E} \prod_{i=1}^{k} f_{i}\left(X_{t_{i}}\right) \geq \prod_{i=1}^{k} \mathrm{E} f_{i}\left(X_{t_{i}}\right) \quad \text { for all } f_{i} \in \mathcal{F}_{i}^{+} .
$$

If (2.1) holds for all $t_{1} \leq \cdots \leq t_{k}$ then we call $X$ associated in time and space, which combines the association in time with the association of $X_{t}$ in space.

Theorem 2.1. (Liggett (1985).) Assume that

(a) $X$ is stochastically monotone, i.e. $f \in \mathcal{F}_{i}^{+}$implies that $T_{t} f \in \mathcal{F}_{i}^{+}$;

(b)

$$
\text { Afg } \geq f A g+g A f \quad \text { for all } f, g \in D_{A} \cap \mathcal{F}_{i}^{+}
$$

and

(c) $\mu=\mathrm{P}^{X_{0}}$ is associated.

Then $X$ is associated in time and space; in particular, $\mu_{t}=\mathrm{P}^{X_{t}}$ is associated for all $t \geq 0$.

Theorem 2.1 was stated in Liggett (1985) for compact partially ordered metric spaces and in Szekli (1995) for products of normally ordered Polish spaces. Stochastic monotonicity in the finite-discrete case has been characterized by Harris (1977) and Cox (1984). The proof of Liggett's result is essentially based on a representation of a solution of a Cauchy problem for $F:[0, \infty) \rightarrow C(E)$ with $F(t) \in D_{A}$ for all $t \geq 0$ and $F^{\prime}(t)=A F(t), F(0)=f \in D_{A}$. In 
the following we derive, in a similar framework to Liggett's theorem, a comparison theorem between two Markov processes with values in a Polish space $E$. The ordering on the set of probability measures $M^{1}(E)$ on $E$ is defined by a cone $\mathcal{F}$ of real-valued functions on $E$ by

$$
\mu \leq_{\mathcal{F}} v \text { if } \quad \int f \mathrm{~d} \mu \leq \int f \mathrm{~d} \nu \text { for all } f \in \mathcal{F} .
$$

Similarly, we define $X \leq \mathcal{F} Y$ for random variables $X$ and $Y$ in $E$. The order generating class is not uniquely defined and typically there are many bounded or smooth and bounded order generating classes. Typical examples of orderings described in this way are the usual stochastic order, various convex orderings, and dependence orderings such as concordance, supermodular, and directionally convex ordering (for definitions and properties, see Müller and Stoyan (2002)).

Let $X$ and $Y$ be homogeneous, strongly continuous Markov processes with values in a Polish space $E$ which have the Feller property. Denote the corresponding semigroups by $S=\left(S_{t}\right)$ and $T=\left(T_{t}\right)$, and the infinitesimal generators by $A$ and $B$ with domains $D_{A}$ and $D_{B}$, respectively. Let $\mathcal{F} \subset C_{b}(E)$ be a cone of bounded, continuous, real functions on $E$, and denote by ' $<\mathcal{F}$ ' the corresponding 'stochastic' order on $M^{1}(E)$. We assume that

$$
\mathcal{F} \subset D_{A} \cap D_{B}
$$

Theorem 2.2. (Conditional ordering result.) Let $X$ and $Y$ be homogeneous Markov processes such that

(a) $X$ is stochastically monotone, i.e. $S_{t} f \in \mathcal{F}$ for all $f \in \mathcal{F}$; and

(b)

$$
\text { Af } \leq B f\left[\mathrm{P}^{X_{0}}\right] \quad \text { for all } f \in \mathcal{F} .
$$

Then

$$
S_{t} f \leq T_{t} f\left[\mathrm{P}^{X_{o}}\right], \quad f \in \mathcal{F} .
$$

Proof. Define, for $f \in \mathcal{F}, F:[0, \infty) \rightarrow C_{b}(E)$ by $F(t):=T_{t} f-S_{t} f$. Then $F(t)$ satisfies the differential equation

$$
\begin{aligned}
F^{\prime}(t) & =B T_{t} f-A S_{t} f \\
& =B\left(T_{t} f-S_{t} f\right)+(B-A)\left(S_{t} f\right) .
\end{aligned}
$$

Note that, by assumption, $S_{t} f \in \mathcal{F}$ and, thus, $H(t):=(B-A)\left(S_{t} f\right)$ is well defined and $H(t) \geq 0$ by (2.4). Thus, $F$ solves the Cauchy problem,

$$
F^{\prime}(t)=B F(t)+H(t), \quad F(0)=0 .
$$

The solution of (2.6) is uniquely determined and is given by (see Liggett (1985, Theorem 2.15) and Szekli (1995, p. 157))

$$
\begin{aligned}
F(t) & =T_{t} F(0)+\int_{0}^{t} T_{t-s} H(s) \mathrm{d} s \\
& =\int_{0}^{t} T_{t-s} H(s) \mathrm{d} s \quad \text { as } F(0)=0 .
\end{aligned}
$$

$H(s) \geq 0$ implies that $F(t) \geq 0$ for all $t$ and, thus, the statement in (2.5) holds. 
Remark 2.3. (a) As mentioned in the introduction, the same idea of proof was used for the case of directionally convex ordering of certain interacting diffusions in Cox et al. (1996) and Greven et al. (2002). Theorem 2.2 can be considered to be a general formulation of this comparison argument.

(b) The notion of generator can be generalized to extended generator allowing for a larger class of not necessarily bounded, continuous functions in their domain $D_{A}$. This is defined by the property that $f \in D_{A}$ if

$$
M_{t}^{f}:=f\left(X_{t}\right)-f\left(X_{0}\right)-\int_{0}^{t} A f\left(X_{s}\right) \mathrm{d} s \in \mathcal{M},
$$

where $\mathcal{M}$ is the class of martingales (see Jacod (1979, Chapter 13)). This property is closely connected to the strong Markov property of $X$. It naturally leads to considering similar ordering properties for the more general class of semimartingales (see Bergenthum and Rüschendorf (2007)).

(c) For several classes of examples, in particular, for Lévy processes, diffusion processes, and jump processes, the propagation of the ordering condition has been studied (see Bergenthum and Rüschendorf (2007)).

Define the componentwise ordering (also called product ordering) of processes $X$ and $Y$ by

$$
(X) \leq \mathscr{F}(Y) \text { if } \operatorname{E} h\left(X_{t_{1}}, \ldots, X_{t_{k}}\right) \leq \mathrm{E} h\left(Y_{t_{1}}, \ldots, Y_{t_{k}}\right)
$$

for all functions $h$ that are componentwise in $\mathcal{F}$. In particular, $(X) \leq \mathscr{F}(Y)$ implies that

$$
X_{t} \leq \mathcal{F} Y_{t} \text { for all } t \geq 0 .
$$

As a consequence of the conditional ordering result in Theorem 2.2 and the separation theorem for the ordering Markov processes (see Bergenthum and Rüschendorf (2007, Proposition 3.1)), we obtain the following ordering result for the processes.

Corollary 2.4. (Comparison result.) If the conditions of Theorem 2.2 hold true and if additionally $X_{0} \leq \mathscr{F} Y_{0}$, then the componentwise ordering $(X) \leq \mathscr{F}(Y)$ of the processes $X$ and $Y$ holds.

\section{Association and applications}

Next we derive Liggett's association result (2.4) as a consequence of Theorem 2.2 and Corollary 2.4 in Section 2. Let $E=\mathbb{R}^{d}$, and let $X=\left(X_{t}\right)$ be a Markov process with values in $E$, as in the introduction. Then $\mu_{t}=\mathrm{P}^{X_{t}}$ is associated if and only if

$$
\left(X_{t}, Y_{t}\right) \leq \mathcal{F}\left(X_{t}, X_{t}\right),
$$

where $Y$ is a conditionally independent copy of $X$, i.e. $Y_{0}=X_{0}, Y$ and $X$ are conditionally independent given $X_{0}$, and $Y \mid Y_{0}=x$ and $X \mid X_{0}=x$ are identically distributed. Furthermore, $\mathcal{F}$ is defined by $\mathcal{F}=\left\{f \otimes g ; f, g \in \mathcal{F}_{i}^{+}\right\}$, where $f \otimes g(x, y)=f(x) g(y)$. Let $\left(S_{t}\right)$ and $A$ respectively denote the semigroup and the infinitesimal generator of $X$ (denoted by $\left.X \sim\left(\left(S_{t}\right), A\right)\right)$. Then $\left(X_{t}, X_{t}\right) \sim\left(\left(\tilde{S}_{t}\right), \tilde{A}\right)$ and $\left(X_{t}, Y_{t}\right) \sim\left(\left(\tilde{T}_{t}\right), \tilde{B}\right)$, where

$$
\begin{gathered}
\tilde{T}_{t} f \otimes g(x, y)=S_{t} f(x) S_{t} g(y), \\
\tilde{S}_{t} f \otimes g(x, y)=S_{t} f g(x), \\
\tilde{B} f \otimes g(x, y)=A f(x) g(y)+f(x) A g(y),
\end{gathered}
$$


and

$$
\tilde{A} f \otimes g(x, y)=\operatorname{Afg}(x) .
$$

For (3.3) and (3.4), we use the assumption that $X_{0}=Y_{0}$.

Corollary 3.1. (Association (Liggett (1985)).) Under conditions (a)-(c) of Theorem 2.1, it holds that $X$ is associated in time and space.

Proof. By Theorem 2.1(a), $X$ is stochastically monotone with respect to $\mathcal{F}_{i}^{+}$and, therefore, for $f \otimes g \in \mathcal{F}$, we find, from (3.1) and (3.2), that $\left(X_{t}, X_{t}\right)$ and $\left(X_{t}, Y_{t}\right)$ are both stochastically monotone with respect to $\mathscr{F}=\mathscr{F}_{i}^{+} \otimes \mathcal{F}_{i}^{+}$. Furthermore, Liggett's condition $A f g \geq f A g+$ $g A f$ for $f, g \in \mathcal{F}_{i}^{+}$implies that

$$
\tilde{A} f \otimes g \geq \tilde{B} f \otimes g\left[\mathrm{P}^{\left(X_{0}, Y_{0}\right)}\right] .
$$

Thus, by Theorem 2.2 we obtain $\tilde{S}_{t} f \otimes g \geq \tilde{T}_{t} f \otimes g\left[\mathrm{P}^{\left(X_{0}, Y_{0}\right)}\right]$, which is equivalent to

$$
S_{t} f g \geq S_{t} f S_{t} g\left[\mathrm{P}^{X_{0}}\right] .
$$

Thus, $X_{t}$ is conditionally associated given $X_{0}$. Condition (c) of Theorem 2.1 and Corollary 2.4 imply that $X$ is associated in time and space.

Remark 3.2. (a) Bäuerle et al. (2006) showed that the Liggett condition (2.2) yields, for the case of Lévy processes, the characterization of association by Samorodnitsky (1995). This characterization states that association of a Lévy process is equivalent to the property that the support of the Lévy measure is contained in the union of the positive and negative orthant of $\mathbb{R}^{d}$, i.e. all jumps are in the same direction.

(b) Theorem 2.2(b) is also a necessary condition for stochastic ordering since, for $f \in \mathcal{F}$, $S_{t} f \leq T_{t} f$ implies that

$$
A f(x)=\lim _{t \downarrow 0} \frac{S_{t} f(x)-f(x)}{t} \leq \lim _{t \downarrow 0} \frac{T_{t} f(x)-f(x)}{t}=B f(x) .
$$

Condition (a) of Theorem 2.2 is in general not a necessary condition.

Example 3.3. In several cases the local comparison condition for the infinitesimal generators is easy to characterize explicitly.

(a) For pure diffusion processes $X$ and $Y$ in $\mathbb{R}^{d}$ with diffusion matrices $\left(a_{i j}\right)=\left(a_{i j}(x)\right)$ and $\left(b_{i j}\right)=\left(b_{i j}(x)\right)$, respectively, the infinitesimal generators are given by

$$
A f(x)=\frac{1}{2} \sum_{i j} a_{i j}(x) \frac{\partial^{2} f}{\partial x_{i} \partial x_{j}} \quad \text { and } \quad B f(x)=\frac{1}{2} \sum_{i j} b_{i j}(x) \frac{\partial^{2} f}{\partial x_{i} \partial x_{j}} .
$$

Thus, for convex ordering, the comparison condition $A f(x) \geq B f(x), f \in \mathcal{F}_{\mathrm{cx}} \cap C^{2}$, is equivalent to

$$
C:=A-B \geq \geq_{\mathrm{psd}} 0,
$$

in the sense of positive semidefiniteness. The stochastic monotonicity needs some strong conditions in dimension $d \geq 2$, while in $d=1$ it is satisfied generally (see Bergenthum and Rüschendorf (2007) for details). Note that the application to convex ordering needs an extension to unbounded functions if the space is not compact. For directionally convex 
ordering ' $\geq_{\mathrm{dcx}}$ ', the corresponding ordering of the infinitesimal generator is given by the more simple comparison

$$
a_{i j}(x) \leq b_{i j}(x) \text { for all } i, j \text { and for all } x .
$$

Cox et al. (1996) and Greven et al. (2002) established, for some class of (infinitedimensional) interacting diffusions, that the stochastic monotonicity condition (as defined in Theorem 2.2) is fulfilled for the case in which $\mathcal{F}=\mathcal{F}_{\mathrm{dcx}}$, the class of directionally convex functions.

(b) For integrable Lévy processes without drift and diffusion, $X \sim(0,0, v)$ and $Y \sim$ $\left(0,0, v^{*}\right)$, where $v$ and $v^{*}$ are the corresponding Lévy measures, the infinitesimal generator are respectively given by

$$
A f(x)=\int_{\mathbb{R}^{d}} \Lambda f(x, y) \mathrm{d} \nu(y) \quad \text { and } \quad A^{*} f(x)=\int_{\mathbb{R}^{d}} \Lambda f(x, y) \mathrm{d} \nu^{*}(y),
$$

where $\Lambda f(x, y)=f(x+y)-f(x)-y \nabla f(x)$. For convex and directionally convex orderings, with respective generating functions $\mathcal{F}_{\mathrm{cx}}$ and $\mathcal{F}_{\mathrm{dcx}}$, the stochastic monotonicity condition is satisfied as $S_{t} f(x)=\int f\left(X_{t}+x\right) \mathrm{d} P$. Thus, we find that the conditions

$$
\begin{aligned}
X_{0} & \leq_{\mathrm{cx}} Y_{0}, & & X_{0} \leq_{\mathrm{dcx}} Y_{0}, \\
v & \leq_{\mathrm{cx}} v^{*}, & & v \leq_{\mathrm{dcx}} v^{*},
\end{aligned}
$$

imply that $X \leq_{\mathrm{cx}} Y$ and $X \leq_{\mathrm{dcx}} Y$. A similar result holds for supermodular ordering ' $\leq_{\mathrm{sm}}$ '. For (3.5), note that we have to pose some integrability condition on $f$. As a consequence of Remark 3.2, this implies that convex, directionally convex, and supermodular orderings of two Lévy processes $X$ and $Y$ are equivalent to the corresponding orderings of the Lévy measures $\mu$ and $\nu$.

A similar conclusion also holds for the stochastic order ' $\leq_{\text {st }}$ ', the upper orthant order ' $\leq$ uo', and the lower orthant order ' $\leq_{10}$ '. Note that upper orthant ordering is generated by the class $\mathcal{F}_{\Delta}$ of $\Delta$-monotone functions (see Rüschendorf (1980)) and, thus, the stochastic monotonicity condition is satisfied for Lévy processes. This is similarly true for lower orthant ordering and, thus, for the combination of both orderings, concordance ordering ' $\leq_{\mathrm{c}}$ '. For the case of supermodular and concordance orderings, this result is stated in Bäuerle et al. (2006) as well as in Bergenthum and Rüschendorf (2007).

The proof of Corollary 3.1 extends to further positive dependence orderings. Let $\mathcal{F}_{\text {ism }}^{+} \subset \mathcal{F}_{i}^{+}$ denote the class of increasing, nonnegative, supermodular functions on $\mathbb{R}^{d}$. Define a random vector $Z=\left(Z_{1}, \ldots, Z_{d}\right)$ to be positive supermodular associated (PSA) if

$$
\mathrm{E} f(Z) \mathrm{E} g(Z) \leq \mathrm{E} f(Z) g(Z)
$$

for all $f, g \in \mathcal{F}_{\text {ism. }}^{+}$. PSA is a weakening of the notion of association. Christofides and Vaggelatou's (2004) association of $Z$ implies positive supermodular dependence, i.e.

$$
Z^{\perp} \leq_{\mathrm{sm}} Z
$$

where $Z^{\perp}$ is a copy of $Z$ with independent components $Z_{i}^{\perp}$ such that $Z_{i}^{\perp} \stackrel{\text { D }}{=} Z_{i}$, where ' denotes equality in distribution. Obviously, the positive supermodular association of $Z$ implies 
positive upper orthant dependence and positive concordance dependence, the combination of positive upper and lower orthant dependence.

Let $\mathcal{F}^{s}$ denote the cone

$$
\mathcal{F}^{s}=\left\{f \otimes g ; f, g \in \mathcal{F}_{\text {ism }}^{+}\right\} .
$$

Then $f, g \in \mathcal{F}_{\text {ism }}^{+}$implies that $f g \in \mathcal{F}_{\text {ism }}^{+}$. Thus, by the representation of the semigroups and generators, as in the case of association (see (3.1)-(3.4)), we obtain the following variant of Corollary 3.1 .

Corollary 3.4. (Positive supermodular association.) Let X be a Markov process as in Section 2 and assume that

1. $X$ is stochastically monotone with respect to $\mathcal{F}_{\text {ism }}^{+}$;

2.

$$
\text { Afg } \geq f A g+g A f \quad \text { for all } f \in D_{A} \cap \mathcal{F}_{\text {ism }}^{+}
$$

and

3. $\mu=\mathrm{P}^{X_{0}}$ is PSA.

Then $X$ is PSA in time and space; in particular, $\mu_{t}=\mathrm{P}^{X_{t}}$ is $P S A$ for all $t \geq 0$.

Remark 3.5. (a) A similar result to Corollary 3.4 also holds if we replace the class $\mathcal{F}_{\text {ism }}^{+}$ by the classes $F_{\text {idcx }}^{+}$of nonnegative, increasing, directionally convex functions or by $\mathcal{F}_{\Delta}^{+}$, the class of (increasing) $\Delta$-monotone functions. As a consequence, we obtain sufficient conditions for positive, increasing, directionally convex dependence and for positive upper orthant dependence. For this conclusion, note that $f, g \in \mathcal{F}_{\Delta}^{+}$implies that $f g \in \mathcal{F}_{\Delta}^{+}$, as can be seen for differentiable $f$ and $g$ by considering $k$ th derivatives. In a similar way we obtain sufficient conditions for positive lower orthant dependence (PLOD) in space. As a consequence, we obtain sufficient conditions for positive concordance dependence. For a discussion of these dependence and ordering concepts, we refer the reader to Müller and Stoyan (2002, Chapter 3.8).

(b) As a particular consequence of Liggett's theorem and Corollary 3.4, consider a Lévy process $X_{t}$ with $X_{1} \stackrel{\mathrm{D}}{=} N(0, \Sigma)$ starting in $X_{0}=0$. Then

$$
A f(x)=\frac{1}{2} \Sigma_{i, j} \sigma_{i j} \frac{\partial^{2} f}{\partial x_{i} \partial x_{j}},
$$

where $\Sigma=\left(\sigma_{i j}\right)$. Liggett's condition (2.2), Afg $\geq(A f) g+f A g$ for $f, g \in \mathcal{F}_{i}^{+}$, is equivalent to

$$
\sum_{i, j} \sigma_{i j}\left(\frac{\partial f}{\partial x_{i}} \frac{\partial g}{\partial x_{j}}+\frac{\partial f}{\partial x_{j}} \frac{\partial g}{\partial x_{i}}\right) \geq 0 \text { for all } f, g \in \mathcal{F}_{i}^{+} \cap C_{b}^{2},
$$

and is thus equivalent to $\sigma_{i j} \geq 0$ for all $i, j$, which is the well-known characterization of association of normal vectors (due to Pitt (1982)). The same condition also holds for the PSA condition (3.6). Thus, for a normally distributed random vector $X \sim N(0, \Sigma)$,

$$
\begin{aligned}
X \text { is associated } & \Longleftrightarrow X \text { is PSA } \\
& \Longleftrightarrow \sigma_{i j} \geq 0 \text { for all } i, j .
\end{aligned}
$$

However, since the PSA dependence is between the association concept and the PLOD, this conclusion is obvious. 


\section{References}

BäUerle, N., Müller, A. And Blatter, A. (2008). Dependence properties and comparison results for Lévy processes. Math. Meth. Operat. Res. 67, 161-186.

Bergenthum, J. And Rüschendorf, L. (2007). Comparison of semimartingales and Lévy processes. Ann. Prob. 35, 228-254.

Chen, M.-F. (2004). From Markov Chains to Non-equilibrium Particle Systems. World Scientific, Singapore.

Chen, M.-F. AND WANG, F.-Y. (1993). On order preservation and positive correlations for multidimensional diffusion processes. Prob. Theory Relat. Fields $\mathbf{9 5}, 421-428$.

Christofides, T. C. AND Vaggelatou, E. (2004). A connection between supermodular ordering and positive/negative association. J. Multivariate Anal. 88, 138-151.

Cox, T. (1984). An alternative proof of a correlation inequality of Harris. Ann. Prob. 12, 272-273.

Cox, T., Fleischmann, K. and Greven, A. (1996). Comparison of interacting diffusions and an application to their ergodic theory. Prob. Theory Relat. Fields 105, 513-528.

Daduna, H. And Szekli, R. (2006). Dependence ordering for Markov processes on partially ordered spaces. J. Appl. Prob. 43, 793-814.

Greven, A., Klenke, A. And Wakolbinger, A. (2002). Interacting diffusions in a random medium: comparison and long-time behaviour. Stoch. Process. Appl. 98, 23-41.

Harris, T. E. (1977). A correlation inequality for Markov processes in partially ordered state spaces. Anna. Prob. 5, $451-454$.

Herbst, I. AND PitT, L. (1991). Diffusion equation techniques in stochastic monotonicity and positive correlations. Prob. Theory Relat. Fields 87, 275-312.

JACOD, J. (1979). Calcul Stochastique et Problèmes de Martingale (Lecture Notes Math. 714). Springer, Berlin.

LiggetT, T. M. (1985). Interacting Particle Systems. Springer, New York.

Massey, W. A. (1987). Stochastic ordering for Markov processes on partially ordered spaces. Math. Operat. Res. 12, 350-367.

Müller, A. And Stoyan, D. (2002). Comparison Methods for Stochastic Models and Risks. John Wiley, Chichester. PITT, L. (1982). Positively correlated normal variables are associated. Ann. Prob. 10, 496-499.

RüsCHENDORF, L. (1980). Inequalities for the expectation of $\Delta$-monotone functions. Z. Wahrscheinlichkeitsth. 54, 341-349.

Samorodnitsky, G. (1995). Association of infinitely divisible random vectors. Stoch. Process. Appl. 55, 45-55.

SzeKLI, R. (1995). Stochastic Ordering and Dependence in Applied Probability (Lecture Notes Statist. 97). Springer, New York. 